\title{
NOVEDADES EN EL GÉNERO STAELIA (RUBIACEAE) PARA ARGENTINA
}

\author{
ELSA L. CABRAL ${ }^{1} \&$ ROBERTO M. SALAS ${ }^{2}$
}

\begin{abstract}
Summary: Cabral, E. L. \& R. M. Salas. 2005. Novelties in the genus Staelia (Rubiaceae) from Argentina. Bonplandia 14(1-2): 83-89. ISSN: 0524-0476.

In Argentina are recognized two species of Staelia: S. thymoides Cham. \& Schltdl. (Corrientes and Entre Ríos) and S. virgata (Roem. \& Schult.) K. Schum. (Jujuy). One new species Staelia juarezii E. L. Cabral \& Salas (W Formosa) and one new variety Staelia virgata var. correntina E. L. Cabral \& Salas (W and central Corrientes) are described and illustrated. The distributions of the former two species is widened.
\end{abstract}

Key words: taxonomy, Rubiaceae, Staelia juarezii, Staelia virgata var. correntina, Argentina.

Resumen: Cabral, E. L. \& R. M. Salas. 2005. Novedades en el género Staelia (Rubiaceae) para Argentina. Bonplandia 14(1-2): 83-89. ISSN: 0524-0476.

En Argentina se conocen dos especies de Staelia: S. thymoides Cham. \& Schltdl. (Corrientes y Entre Ríos) y S. virgata (Roem. \& Schult.) K. Schum. (Jujuy) a las que se agrega una nueva especie S. juarezii E. L. Cabral \& Salas (oeste de Formosa) y una nueva variedad S. virgata var. correntina E. L. Cabral \& Salas (oeste y centro de Corrientes). Se amplía la distribución de las dos primeras especies.

Palabras clave: taxonomía, Rubiaceae, Staelia juarezii, Staelia virgata var. correntina. Argentina.

El género Staelia Cham. \& Schltdl., es exclusivamente sudamericano y fue descripto en 1828 sobre una especie del sur de Brasil, $S$. thymoides. Pertenece a la tribu Spermacoceae y se diferencia de los géneros vecinos por la dehiscencia transverso-oblicua del fruto, que se separa en tres partes, dos apicales y una basal persistente. Cuenta con cerca de 14 especies (Andersson, 1992; de Souza \& de Sales, 2004), la mayor parte de las cuales habitan en Brasil.

En Argentina se han registrado 2 especies,
Staelia thymoides Cham. \& Schltdl. y S. virgata (Willd. ex Roem. \& Schult.) K. Schum. (Bacigalupo \& Cabral, 1999), a las que se agrega una nueva especie, $S$. juarezii $\mathrm{E}$. L. Cabral \& Salas, y una nueva variedad $S$. virgata var. correntina E. L. Cabral \& Salas.

Sobre el género Staelia se conocen hasta el presente sólo estudios parciales o contribuciones en floras regionales (Schumann, 1889; Chodat \& Hassler, 1904; Bacigalupo, 1974, 1993; Porto \& al., 1977; de Souza \& de Sales, 2004). La mayor parte de las especies conoci-

\footnotetext{
${ }^{1}$ Instituto de Botánica del Nordeste, UNNE-CONICET, C. C. 209, 3400. Corrientes, Argentina. Fac. Ci. Exact. y Nat. y Agrim. UNNE, e-mail: ecabral@agr.unne.edu.ar; ferber@arnet.com.ar

${ }^{2}$ Becario de Pregrado SGCyT-UNNE, e-mail: robertoymanuels@hotmail
} 
das fueron descriptas por Schumann (1889), Standley (1931), Sucre (1959, 1971), Kirkbride (1997) y Cabral \& Bacigalupo (2005).

\section{Staelia Cham. \& Schltdl.}

Chamisso, L. A. \& D. F. L. Schlechtendal, Linnaea 3: 364, tab.3. 1828. Especie tipo: Staelia thymoides Cham. \& Schltdl.

Hierbas perennes, rara vez anuales. Hojas a menudo lineares, estípulas envainadoras, fimbriadas. Flores pequeñas, en glómerulos sésiles, axilares y terminales. Cáliz de 2 sépalos persistentes. Corola hipocrateriforme o infundibuliforme, 4-lobada. Estambres 4, fijos en la garganta de la corola, exertos. Ovario 2-locular; estilo filiforme, de ápice bífido o bilobado. Fruto capsular, de dehiscencia transverso-oblicua en cada carpelo, parte basal entera, con tabique intercarpelar persistente, mitades apicales independientes y caducas; semillas surcadas en la cara ventral.

\section{Clave para diferenciar las especies y variedades argentinas de Staelia}

1. Glomérulo apical y 2 (3) axilares, de desarrollo basítono, el apical de mayor diámetro (con frecuencia glomérulo apical y subapical muy próximos, aparentando tener involucro de 4 brácteas). Disco nectarífero bilobado. Vaina estipular con 3 (-5) lacinias. Argentina (centro y este de Corrientes, este de Entre Ríos, Misiones), Brasil (Rio Grande do Sul), Paraguay (sur de Paraguay Oriental) y oeste de Uruguay.

\section{S. thymoides Cham. \& Schltdl.}

1'.Glomérulo apical y (4-) 6-20 (-30) axilares. Glomérulos con desarrollo acrópeto, el apical de menor diámetro, involucro de 2 brácteas. Disco nectarífero entero o bipartido. Vaina estipular con 3-5 (-8) lacinias.

2. Corola 1,5-1,8 mm long., de largo casi igual al del cáliz. Estambres y estilo más cortos que la corola. Interior de la corola con pelos dispersos hasta la base de los lóbulos. Disco nectarífero entero. Argentina (oeste de Formosa).

1. S. juarezii E. L. Cabral \& Salas

2'.Corola 5-8 (-9) mm long., notablemente más larga que el cáliz. Estambres y estilo iguales o más largos que la corola. Interior de la corola con anillo de pelos moniliformes \pm en la mitad inferior del tubo. Disco nectarífero entero o bipartido.

3. Glomérulos de 10-15 mm lat., 6-20 (-30) por rama florífera. Corola 5-6 (-6,5) mm long., externamente glabrescente a hírtula. Disco entero. Valvas caducas del fruto densamente hírtulas a pubescentes en toda su superficie. Vaina estipular pilosa o pubescente con 3-5 (-8) lacinias. Planta robusta de 30-100 cm alt. Argentina (Jujuy, Salta, S del Estero, Tucumán, Chaco, Formosa), Bolivia, Brasil, Paraguay y Perú.

2a. S. virgata (Willd. ex Roem. \& Schult.) K. Schum. var. virgata

3'.Glomérulos de 3-10 (-15) mm lat., 4-10 (-12) por rama florífera. Corola (6-) 7-8 (-9) mm long., externamente pubescente. Disco bipartido. Valvas caducas del fruto pubescente sólo en el tercio superior. Vaina estipular glabra o glabrescente, con $3(-5)$ lacinias. Planta de 10-30 (-41) cm alt. (oeste y centro de Corrientes). 
E.L. Cabral \& R.M. Salas, Novedades en el género Staelia (Rubiaceae)

\section{Staelia juarezii E. L. Cabral \& Salas sp. nov.}

Fig. 1

Suffrutex 30-40 cm altis, ramosus, caulis pubescentis; folia 10-15 mm longa x 0,3-0,5 mm lata, pseudofasciculata; glomeruli 10-15 (-18) in rami florale, 3-4 (-5) $\mathrm{mm}$ latis, 2 bracteae involucri, corolla 1,5-1,8 mm longa.

Holotypus: ARGENTINA. Formosa: Dep. Matacos, $13 \mathrm{~km} \mathrm{~S}$ de Ing. Guillermo N. Juárez, comunidad flechillar sacha alfarfar, 11-VI-1972, fl fr, R. Burkart \& al. s. n. (SI).

Sufrútice 30-40 $\mathrm{cm}$ alt., ramificado desde la base. Tallos subcilíndricos, fistulosos, pubescentes, pelos hialinos, adpresos, antrorsos, entrenudos 5-18 mm long. Hojas 10-15 $\mathrm{mm}$ long. $\mathrm{x}$ 0,3-0,5 $\mathrm{mm}$ lat., pseudoverticiladas, lineares o linear-lanceoladas, apiculadas, glabras, sólo cerca de la base con pelos similares al tallo. Vaina estipular $0,5-1 \mathrm{~mm}$ long., glabrescente, con 3-5 lacinias de 0,2-3 (-4) mm long. Glomérulos 10-15 (-18) por rama florífera, 3-4 (-5) $\mathrm{mm}$ lat., brácteas involucrales 2. Hipanto 0,6-0,9 mm long., turbinado, híspido en la mitad superior; segmentos del cáliz linear-subulados, erguidos, 1,2-1,4 mm long., híspidos en la base; corola 1,5-1,8 mm long., superficie externa hispídula, superficie interna con pelos dispersos desde \pm mitad del tubo hasta la base de los lóbulos; estambres más cortos que los lóbulos, anteras 0,4-0,5 mm long.; estilo filiforme, 1,61,7 mm long., estigma bilobado. Disco entero. Cápsula híspida en las valvas y con pocos pelos en la base persistente. Semilla subelipsoide, 0,7-0,9 mm long., verrucosa, con los surcos bordeando el estrofíolo prolongados hasta el borde inferior.

Distribución geográfica: Vive en el oeste de Formosa. Suelo arenoso-arcilloso en comunidades de Schinopsis lorentzii (Griseb.) Engl. y Aspidosperma quebracho-blanco Schltdl.

Paratypus: ARGENTINA. Formosa: Dep. Matacos, 15 km S de Ing. Guillermo N. Juárez, 11VI-1972, fl, Schulz 18233 (CTES).

\section{2a. Staelia virgata (Willd. ex Roem. \& Schult.) K. Schum. var. virgata}

Schumann, K., in Mart., Fl. bras. 6 (6): 76. 1888.

Spermacoce virgata Willd. ex Roem. \& Schult., Syst. veg. 3: 281, 531. 1818. Typus: Brasil, Hoffmannsegg (B-WILLD 2634)!

Mitracarpum virgatum (Roem. \& Schult.) Cham. \& Schltdl., Linnaea 3: 363. 1828.

Staelia caespitosa Griseb., Symb. fl. argent.: 158. 1879. Foto syntypus F 898.

Distribución geográfica y hábitat: Staelia virgata var. virgata es el taxón de más amplia distribución de todas las especies del género. En Brasil, excepto en los estados del sur, vive en casi todo el país (Amapá, Bahía, Ceará, Goiás, Maranhão, Mato Grosso, Minas Gerais, Nordeste, Pará, Paraíba, Pernambuco, Piauí, Tocantins), habita también en Bolivia, Paraguay y Perú. En Argentina fue citada para la provincia de Jujuy (Bacigalupo \& Cabral, 1999), en este trabajo se agregan ejemplares de Salta, Tucumán, Santiago del Estero, Chaco y oeste de Formosa. Habita el Chaco seco en suelos arenoso-arcillosos y en el borde de las Yungas entre 500-1100 m.

Material estudiado: ARGENTINA. Chaco: Dep. Güemes, Nueva Pompeya, 13-III-1970, Schulz 17425 (CTES). Formosa: Dep. Bermejo, Pozo de Maza, 2334'S, $61^{\circ} 43^{\circ} \mathrm{W}, 26-\mathrm{I}-1986$, Maranta 963 (BACP, CTES); Dep. Matacos, Ruta 39, $23 \mathrm{~km} \mathrm{~S}$ de Ing. G. Juarez, camino a Río Bermejo, 26-XI-1994, Krapovickas \& Cristóbal 46387 (CTES); ídem, 6-III-2001, Schinini \& al. 35331 (CTES). Jujuy: Dep. San Pedro, El Milagro, 24-I-1976, Cabrera \& al. 27511 (CTES, SI). Salta: Dep. Anta, El Quebrachal, 13-II-1947, Luna 695 (CTES, LIL); Pozo largo, 12-XI-1987, Saravia Toledo 1447 (CTES); Dep. La Capital, San Luis, 11-I-1949, Filipovich 394 (CTES, LIL); Alvarado, 20-I-1949, Filipovich 287 (CTES, LIL); $3 \mathrm{~km}$ de Salta, camino a San Lorenzo, La Loma, 10-IV-1980, Krapovickas \& al. 35968 (CTES); Cerro San Bernardo, II-1936, Schulz 1453 (CTES); Dep. General Güemes, Juramento, 22-III-1977, Krapovickas \& al. 30440 (CTES); Arroyo Garrapatas, 14-XII-1989, Novara \& al. 9173 (CTES); Yaquiasmé, 8-II-1996, Novara 10799 (CTES); 


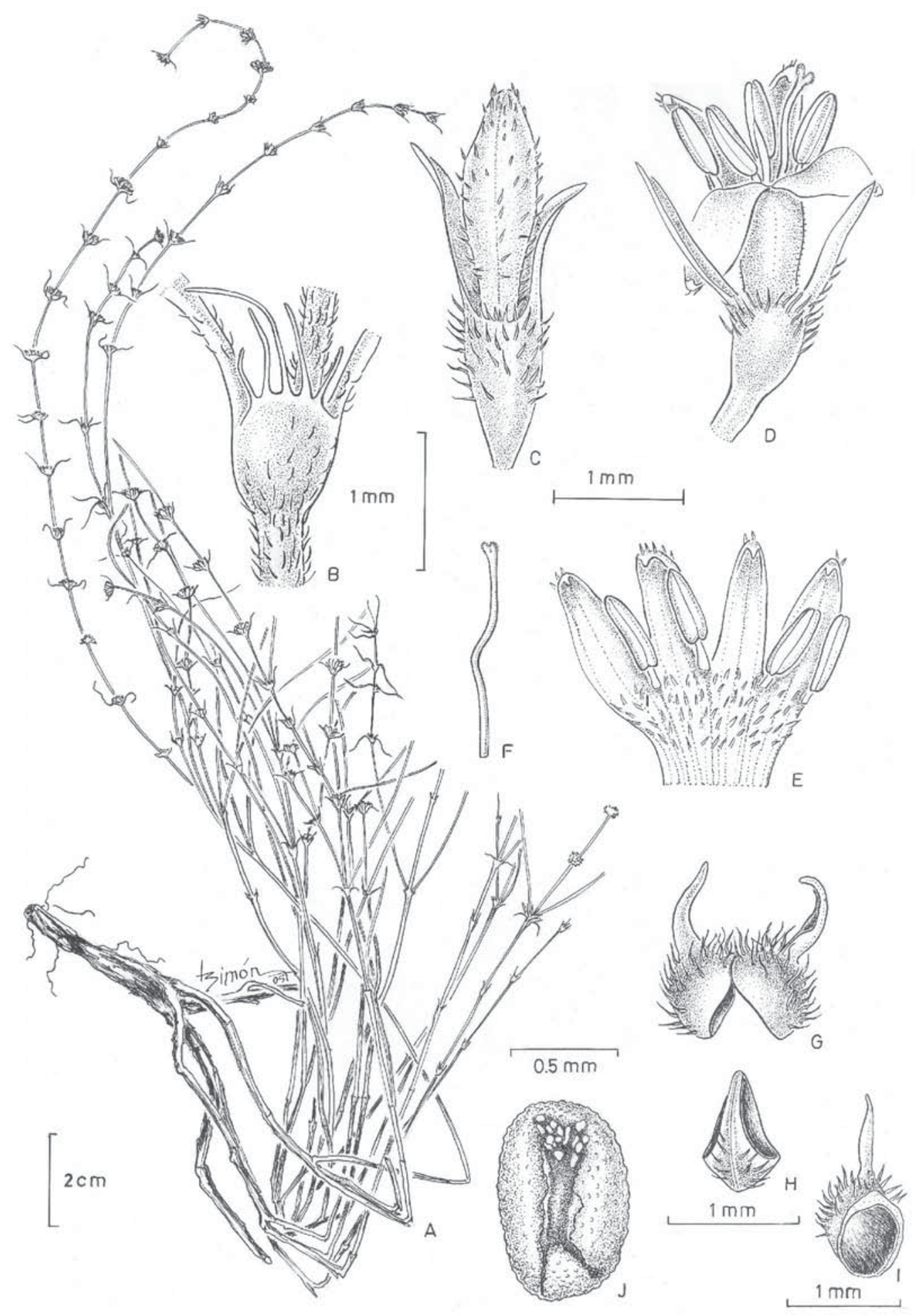

Fig.1. Staelia juarezii. A: planta. B: vaina estipular con lacinias. C: alabastro. D: flor madura. E: interior de la corola desplegada. F: estilo y estigma. G-I: fruto dehiscente. G, mericarpos caedizos aún no separados entre sí. H: parte basal. I: mericarpo caduco, cara interna. J: semilla, cara ventral. (R. Burkart \& al. s. n). Dibujó Laura Simón. 
Dep. General José de San Martín, Coronel Cornejo, 9-XII-1972, Maruñak \& al. 591 (CTES); Dep. Metán, Juramento, 4-IV-1945, O’Donnell 2712 (CTES, LIL); Km 1374, 26-III-1969, Meyer \& al. 9193 (CTES, LIL); Lumbreras, 9-XII-1976, Lotti 67 (CTES, LIL); Dep. Orán, 2 km al Sur de Orán, 10-XII-1998, Saravia Toledo 14563 (CTES); Dep.

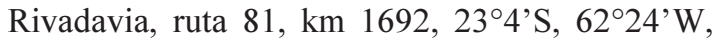
15-I-2002, Solís Neffa \& al. 596 (CTES); $10 \mathrm{~km} \mathrm{E}$ de Los Blancos, 7-V-1998, Krapovickas \& al. 47249 (CTES); 16.5 km W de Capitán Page, 7-III2001, Schinini \& al. 35367 (CTES). Santiago del Estero: Dep. Robles, Vilmer, 31-X-1946, Ruiz de Huidobro 3072 (CTES, LIL); Dep. Banda, 17 km al NO de la Banda, rumbo al Dique Los Quiroga, 13-XI-1971, A. T. Hunziker \& al. 21584 (CTES). Tucumán. Dep. Burruyacu, Sierra Nogalito, 2-II1963, Krapovickas \& al. 10894 (CTES, LIL).

\section{2b. Staelia virgata (Willd. ex Roem. \& Schult.) K. Schum. var. correntina E. L. Cabral \& Salas var. nov.}

Fig. 2

Suffrutex 10-30 (-41) cm altus; glomeruli 4-10 (-12) in rami florale, corolla (6-) 7-8 (-9) $\mathrm{mm}$ longa; discus bipartitus; capsula, valvae supra pubescentia.

Typus: ARGENTINA. Corrientes: Dep. Capital, Riachuelo, borde de arroyo, 11-VI-1972, fl fr, R. M. Salas 170 (holotypus: CTES, isotypi: PEUFR, SI).

Sufrútice 10-30 (-41) cm alt., tallos cilíndricos, raro subtetrágonos, con cortos pelos curvos, antrorsos. Hojas opuestas, pseudoverticiladas, 15-35 mm long. x 1-4 mm lat., lineares a angostamente elípticas, agudas, apiculadas, glabras en ambas caras, excepto en la base con pubescencia similar al tallo; vaina estipular con $3(-5)$ lacinias, glabra o glabrescente y con escasos pelos cerca del borde. Glomérulos 4-10 (-12), de 3-10 mm lat., en cada rama florífera. Hipanto 0,8-1 mm long., cáliz con segmentos linear-subulados, erguidos, pilosos, 2-2,5 mm long.; corola (-6) 7-8 (-9) mm long., pubescente por fuera y sólo con anillo de pelos moniliformes en su inte- rior \pm a la mitad del tubo corolino; estambres exsertos, 1-1,3 mm long.; estilo exserto, 8-9 $\mathrm{mm}$ long., estigma bífido, 0,5-0,8 mm long. Disco bipartido. Cápsula de valvas pubescentes en el tercio superior; semilla subelipsoide, 1-1,2 mm long., reticulado-foveolada, con surco ventral rodeando el estrofíolo persistente.

Distribución geográfica y hábitat: Argentina, oeste y centro de Corrientes. Vive principalmente en suelos arenosos o pedregosos, en bordes de ríos y arroyos.

Material estudiado: ARGENTINA. Corrientes: Dep. Bella Vista, Bella Vista, alrededores del puerto, 25-III-1979, J. Irigoyen 482 (CTES); ídem, 19-XI-2003, Cocucci \& al. 3051 (CORD, CTES); $10 \mathrm{~km} \mathrm{~S}$ de Bella Vista, 8-XI-1978, Schinini \& Ahumada 15903 (CTES); Dep. Capital, Riachuelo, 15-II-96, Schinini 30431 (CTES); Camba Punta, barranca río Paraná, 18-IX-1963, Schulz 12313 (CTES, SI); Dep. Empedrado, Paraje Costa Mansión, 28-III-1994, Baldo 13 (CTES); Dep. Saladas, Colonia Tatacuá, 18-XI-93, Schinini 1204 (CTES); Ea. "La Amistad", 9-II-1950, Schwarz 9644 (CTES); Dep. Mercedes, sobre ruta 123, $10 \mathrm{~km}$ SE de Mercedes, 29-X-1980, Cáceres 45 (CTES); Dep. San Cosme, Barranca del río Paraná, entre Corrientes y Paso de la Patria, 9-X1963, Schulz 12397 (CTES); Dep. San Roque, Ruta 17 y camino a Pando, 13-I-1971, Carnevali 2398 (CTES); Ea. Tatacuá, 18-XII-1970, Carnevali 2260 (CTES).

\section{Staelia thymoides Cham. \& Schltdl.}

Chamisso, L. A. \& D. F. L. Schlechtendal, Linnaea 3: 364. 1828. Typus: S Brazil "In Brasilia meridionali lectam misit Sellow" (B, destruido).

Observaciones: Por la falta de revisión del género Staelia, aún se encuentran confusiones en las identificaciones de sus especies y $S$. thymoides es el nombre más usado en los herbarios sobre material erróneamente identificado. Se comprueba su distribución geográfica restringida al sur de Brasil (Rio Grande do Sul), Argentina (Misiones, centro y este de Corrientes, este de Entre Ríos), oeste del Uruguay y sur de Paraguay Oriental. 


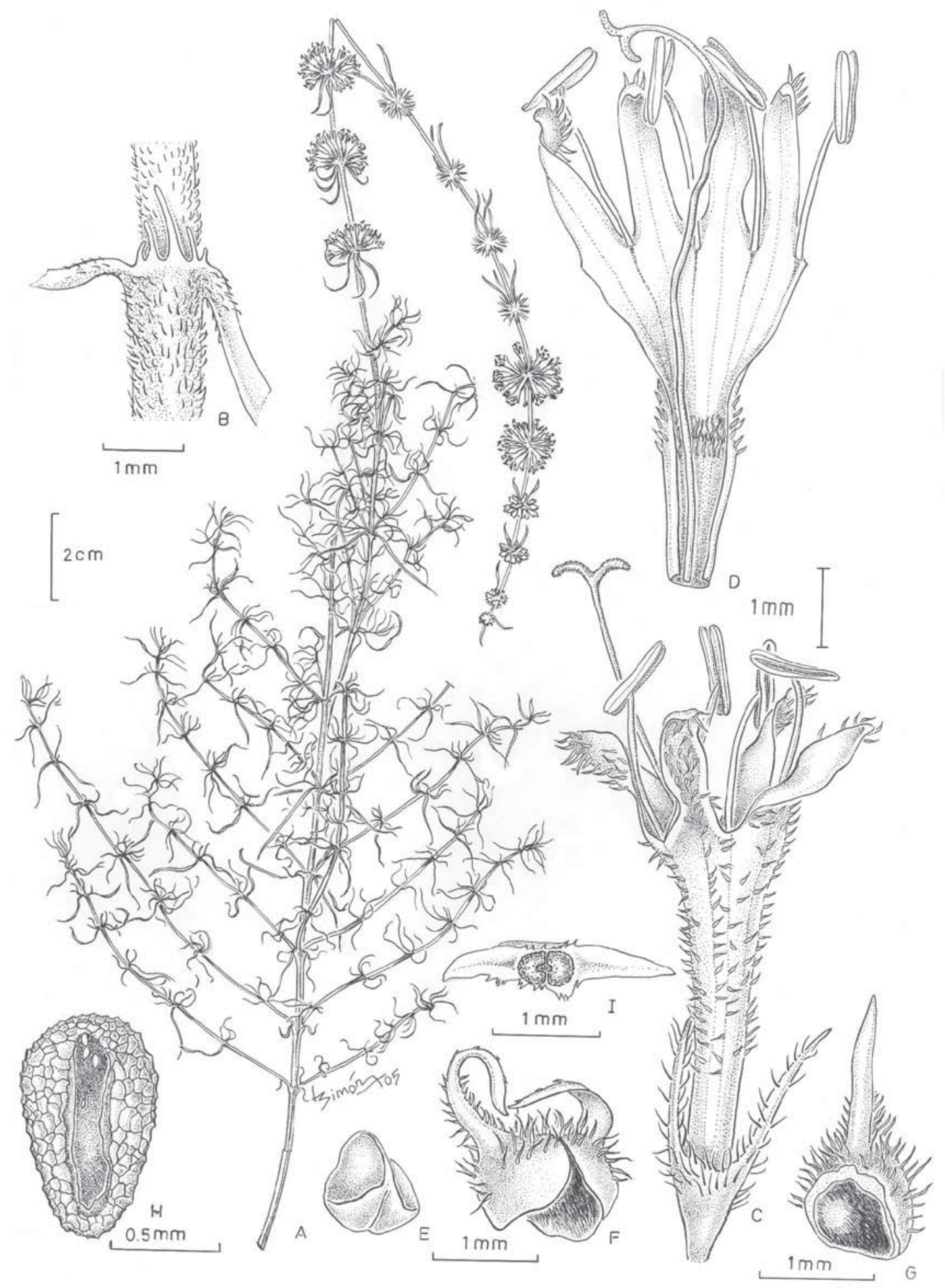

Fig. 2. Staelia virgata var. correntina. A: rama. B: vaina estipular con lacinias. C: flor. D: interior de la corola desplegada. E-G: fruto dehiscente. E: parte basal entera. F: mericarpos caducos aún no separados entre sí. G: mericarpo, segmento caduco, cara interna. H: semilla, cara ventral. I: disco nectarífero bipartido y cáliz, vista apical (Salas 170). Dibujó Laura Simón. 
Material estudiado: ARGENTINA. Corrientes: Dep. Ituzaingó, Ruta 39 y Río Aguapeí, 22-II1980, Cabral 168 (CTES); Dep. Mercedes, Destacamento de la Reserva Nacional Iberá, 5-IX-1997, Ferrucci 1273 (CTES); Dep. Monte Caseros, Juan Pujol, 9-II-1945, Ibarrola 2351 (CTES, LIL); Dep. Paso de los Libres, Paso de los Libres, Laguna Mansa, 30-X-1973, Schinini 7598 (CTES); Dep. Santo Tomé, Caza Pava, 21-XI-1944, Ibarrola 1389 (CTES, LIL); Dep. San Martín, Yapeyú, 29IV-1987, Sáenz \& Morrone 323 (CTES). Entre Ríos: Dep. Colón, Parque nacional El Palmar, camino a Prefectura, 14-III-1981, Cusato 608 (BAA, CTES); Dep. Concordia, Estación Agronómica, Burkart 1109 (SI); Dep. Federación, Federación, 20-XII-1946, Meyer 11121 (CTES, LIL). Misiones: Dep. Candelaria, Loreto, 20-I-1972, Krapovickas \& al. 20776 (CTES); Dep. Cainguás, ruta 14 km, 252, 24-I-1950, Schwindt 3046 (CTES, LIL); Dep. Capital, Posadas, 30-III-1945, Bertoni 1128 (CTES, LIL); Dep. Eldorado, Eldorado, I-X-1949, Schwindt 2111 (CTES, LIL); Dep. San Ignacio, Teyucuaré, 14-II-1980, Schinini 19861 (CTES); Dep. San Pedro, Arroyo Liso, 18IX-1945, Bertoni 1928 (CTES, LIL). BRASIL. Rio Grande do Sul: Uruguaiana, 21-V-1968, Krapovickas 14339 (CTES); São Borja, 4-XII1973, Waechter 1975 (CTES, ICN); Quarai, 21XI-1973, Detoni 1973 (CTES, ICN); Itaquí, 5-IV1975, Porto 1453 (CTES, ICN); Rosario do Sul, 20-I-1973, Krapovickas \& al. 22813 (CTES). PARAGUAY. Central: Villeta, 16-XI-1969, Pedersen 9331 (CTES). Cordillera: $13 \mathrm{~km}$ E del camino Piribebuy-Paraguarí, 2-III-1994, Krapovickas \& al. 45168 (CTES). Caaguazú: Coronel Oviedo, 5II-1985, Bordas 3603 (CTES). Paraguarí: Salto Piraretá, III-1972, Schinini 4353 (CTES). URUGUAY. Artigas: $3 \mathrm{~km}$ del desvío a T. Gomensoro, 10-XII-2005, Solís Neffa \& al. 229 (CTES).

\section{Bibliografía}

ANDERSSON, L. 1992. A provisional checklist of Neotropical Rubiaceae. Scripta Bot. Belg. 1: 1-199.
BACIGALUPO, N. M. 1974. Rubiaceae. In A. Burkart (ed.). Fl. Il. Entre Ríos Colecc. Ci. Inst. Nac. Tecnol. Agropecu. 6 (6):30-32.

- 1993. Rubiaceae. In A. L. Cabrera (ed.). Fl. Prov. Jujuy. Colecc. Ci. Inst. Nac. Tecnol. Agropecu. 13 (9): 420-422.

BACIGALUPO, N. M. \& E. L. CABRAL. 1999. Staelia (Rubiaceae). In F. O. Zuloaga \& O. Morrone (eds.). Catálogo de Plantas Vasculares de la República Argentina. Monogr. Syst. Bot. Missouri Bot. Gard. 74: 1014.

CABRAL, E. L. \& N. M. BACIGALUPO. 2005. Novelties in Spermacoceae (Rubiaceae) from Bolivia and Paraguay. Brittonia 57 (2): 129-140.

CHODAT, R. H. \& E. HASSLER. 1904. Plantae Hasslerianae soit énumération des plantes récoltées au Paraguay par le Dr Émile Hassler d'Aarau (Suisse) de 1885 à-1902 et publiées par le Prof. Dr R. Chodat et le Dr E. Hassler. Seconde partie. Rubiaceae. Bull. Herb. Boissier ser. 2 (4): 90-92, 169-192.

CHAMISSO, L. A. \& D. F. L. SCHLECHTENDAL. 1828. De Plantis in expeditione speculatoria Romanzoffiana abservatis. Linnaea 3 (4): 338-366.

DE SOUZA, E. B. \& M. FERREIRA DE SALES. 2004. O gênero Staelia Cham. \& Schltdl. (RubiaceaeSpermacoceae) no Estado de Pernambuco, Brasil. Acta Bot. Bras. 18 (4): 919-926.

KIRKBRIDE, J. H. 1997. Manipulus rubiacearum VI. Brittonia 49: 376-378.

PORTO, M. L., S. M. C. JACQUES, S. T. MIOTTO, J. L. WAECHTER \& M. L. DETONI. 1977. Tribo Spermacoceae Rubiaceae I. Fl. Il. Río Grande do Sul 35 (5): 82-85.

SCHUMANN, K. 1889. Rubiaceae. In C. P. F. Martius (ed.), Fl. bras. 6 (6): 71-76.

STANDLEY, P. 1931. Studies of American Plants-V., Field Mus. Nat. His., Bot. Ser. 8 (5): 295-398.

SUCRE BENJAMIN, D. 1959. Rubiaceae da cidade do Rio de JaneiroI-Tribo Spermacoceae. Rodriguésia 33-34:241-262.

—. 1971. Estudo das Rubiaceae Brasileiras, III: Cinco novas espécies da tribo Spermacoceae. Rodriguésia 38: 253-260.

Original recibido el 23 de mayo de 2005; aceptado el 6 de julio de 2005. 